\title{
Zolpidem Dependency and Withdrawal Seizure: A Case Report Study
}

\author{
Seyed Alireza Haji Seyed Javadi ${ }^{1}$; Farid Hajiali ${ }^{2}$; Marjan Nassiri-Asl ${ }^{3,}$ \\ ${ }^{1}$ Department of Psychiatry, Faculty of Medicine, Qazvin University of Medical Sciences, Qazvin, IR Iran \\ ${ }^{2}$ Department of Psychiatry, Faculty of Medicine, Qazvin University of Medica \\ ${ }^{3}$ Department of Pharmacology, Faculty of Medicine, Qazvin University of Medical Sciences, Qazvin, IR Iran \\ ${ }^{*}$ Corresponding Author: Marjan Nassiri-Asl, Department of Pharmacology, Faculty of Medicine, Qazvin University of Medical Sciences, Qazvin, IR Iran. Tel: +98-2813336001, E-mail: \\ marjannassiriaslm@gmail.com
}

Received: May 8, 2014; Revised: September 17, 2014; Accepted: October 6, 2014

\begin{abstract}
Introduction:Zolpidem is a short acting inducer of sleepand thought tolack benzodiazepine properties such as anxiolysis, anticonvulsion, muscle relaxation and side effects such as dependency. Recently, some cases of Zolpidem abuse and dependency have been reported. In review of literature, we found that the lowest reported dosage of Zolpidem, which caused dependency, was 160 mg daily.

Case Presentation: We reported a 30-year-old unmarried Iranian woman with dysthymic disorder and chronic insomnia treated with Zolpidem irregularly. She started to use Zolpidem with $5 \mathrm{mg}$ per day irregularly since a year ago but augmented its daily dosage gradually to 100 to $150 \mathrm{mg}$ per day in divided doses. After a period of 16 hours without taking Zolpidem she developed a withdrawal syndrome, with generalized tonic-clonic seizures for two times. She was managed with supportive care and recovered completely.

Conclusions: Zolpidem dependency and withdrawal seizure can occur with a dosage under last reported doses. Therefore, possibility of mentioned problems cannot be excluded at any dosage and physicians should pay more attention to potential of Zolpidem to create these adverse effects.
\end{abstract}

Keywords:Zolpidem; Dependence; Benzodiazepine

\section{Introduction}

Zolpidem (an imidazopyridine derivative agent) is a nonbenzodiazepine hypnotic drug with a high affinity to $\alpha 1$ subunit of gamma amino butyric acid-A (GABA-A) receptor and minor anxiolytic and anti-convulsant effects which is indicated for short-term management of insomnia (1). Zolpidem is thought to be a safer drug than benzodiazepines (BZD) because of no evidence of abuse or dependence potential and a less liability for abuse and dependence (2). Against so many studies indicating no evidence regarding abuse or dependence potential by Zolpidem, case reports of Zolpidem abuse or dependence (3-5) and epileptic-seizure related to Zolpidem withdrawal (6-8) are increasing. To our knowledge, most of these case reports have been reported from Western countries (9) and in the Asian population, one case of Zolpidem dependence (10) and one case of Zolpidem withdrawal seizure (6) were reported. Nonetheless, in Iranian people, we did not find any similar report. Moreover, withdrawal seizure in our case with 100 to $150 \mathrm{mg} /$ day of Zolpidem is the minimum dosage reported up to now.

\section{Case Presentation}

On October 2013, a 30 year-old unmarried Iranian woman (known case of dysthymic disorder) was admitted to Emergency Department (ED) of 22-Bahman Psychiatric Hospital (Qazvin, Iran) with seizure without any history of head trauma. No medications were administered en route to the hospital. For about five minutes early after admission, she had seizure one time again, thus she suddenly had tonic-clonic seizure (full body "shaking" movements lasting approximately two minutes) with upward gaze and loss of consciousness. Then, postictal confusion with clouded consciousness, regressed attitude and behavior and psycho-motor retardation happened for about two hours. After postictal phase, she indicated to use Zolpidem for a year due to insomnia and not receiving any other medication. She started to use Zolpidem with 5 mg per day irregularly since a year ago but augmented its daily dosage gradually from three months before to 100$150 \mathrm{mg}$ per day in divided doses. She used this dosage for about one month prior to her seizure. She had drug tolerance, abuse and dependence and if she had not used tablets, she would become irritable with decreased energy, feeling of weakness and tremor of hands and feet. In the day of admission, she had not used Zolpidem to maintain her alertness for an important ceremony and after a period of 16 hours without taking Zolpidem, she developed an abstinence syndrome, with generalized tonic clonic seizures. In her medical history, she did not have any systemic, organic, metabolic or endocrine problems unless a history of adenoidectomy 25 years ago and dysthymic disorder from one year ago. She had not experienced any seizure already. In her drug history, she just had used Zolpidem with the mentioned dosage. Some of patient's characteristics were summarized in Table 1.

Copyright ( ) 2014, Iranian Red Crescent Medical Journal. This is an open-access article distributed under the terms of the Creative Commons Attribution-NonCommercial 4.0 International License (http://creativecommons.org/licenses/by-nc/4.0/) which permits copy and redistribute the material just in noncommercial usages, provided the original work is properly cited. 
Haji Seyed Javadi SA et al.

\begin{tabular}{lc}
\hline Table 1. Some Laboratory and Clinical Results of Patient \\
\hline & Results \\
\hline Oral temperature, ${ }^{\circ} \mathbf{C}$ & 36.8 \\
\hline Heart rate, beats/min & 106 \\
Respiratory rate, breaths/min & 23 \\
\hline Systolic blood pressure, $\mathbf{m m H g}$ & 128 \\
\hline Diastolic blood pressure, $\mathbf{m m H g}$ & 78 \\
Percutaneous $\mathbf{O}_{\mathbf{2}}$ saturation, \% & 99 \\
\hline Serum glucose, $\mathbf{m g} / \mathbf{d L}$ & 112 \\
\hline
\end{tabular}

Her pupils were $4 \mathrm{~mm}$ and reactive bilaterally. The initial resting 12 lead electrocardiography (EKG) showed normal sinus rhythm without any abnormal changes. There was no localizing or lateralizing neurological signs. Full blood count, urea, electrolytes, calcium, magnesium, hematology studies, renal, thyroid and liver function tests had normal results. Substance-drug abuse tests had negative results. Due to the urgency of patient and availability of computed tomography (CT) scan, we first performed spiral brain CT scan without contrast, which had normal findings. The next day we requested brain magnetic resonance imaging (MRI) and electroencephalography (EEG), which had normal findings. After all evaluations, we did not find any other etiologies except Zolpidem withdrawal. Our patient was detoxified by tapering Zolpidem gradually over one week. We prescribed quetiapine $25 \mathrm{mg}$ before sleep and clonazepam $1 \mathrm{mg}$ per day. No other seizure attack was noted during hospitalization. Finally, after about 10 days hospitalization, she was discharged with a healthy condition with venlafaxine $75 \mathrm{mg}$ thrice daily (t.i.d), clonazepam $1 \mathrm{mg}$ daily and quetiapine $25 \mathrm{mg}$ daily at bed time (for her insomnia). In about 6 months follow-up after the first seizure, she had no further seizure attacks.

\section{Discussion}

During the last decade, Zolpidem (a non BZD hypnotic drug) was considered a new way for treatment of patients with insomnia as it was suggested that it has the efficacy of BZDs for insomnia but without many side effects. It was suggested that Zolpidem lacked muscle relaxant, anticonvulsant and anxiolytic properties and poor potential for abuse or dependence (11). GABA-A receptors include $\alpha_{1}, \alpha_{2}, \alpha_{3}, \alpha_{4}$, and $\alpha_{5}$ subunits receptors. The $\alpha_{1}$ subunit involves in sleeping mechanisms and $\alpha_{2}$ subunit contributes to anxiolytic action. BZDs have nonselective affinity to GABA-A subunits (12). Despite the fact, Zolpidem has been suggested to have selective activity on $\alpha 1$ subunit, but low affinity for $\alpha_{2}$, therefore it has minor anxiolytic action. Our patient reported anxiolysis after using Zolpidem. It might be due to effects of this drug in high doses (such as that used by our patient) not only on $\alpha_{1}$ subunit, but also on other subunits of GABA-A receptors (leading to an anxiolytic effect). In some cases, Zolpidem has been used to achieve euphoria and stimulation and not for sedation $(3,13,14)$. Since this effect lasted not more than one hour, they repeated the intake in the daytime. A hypothesis about Zolpidem withdrawal is long-term supratherapeutic doses saturation of the lower-affinity $\alpha_{2}, \alpha_{3}$ and $\alpha_{5}$ subunits on GABA-A receptors along with $\alpha_{1}$ subunits (15). Therefore, high-dose Zolpidem may have a paradoxical effect to decrease anxiety, and abrupt discontinuation of high doses would produce withdrawal symptoms such as anxiety, tremor, palpitation, or seizure (similar to BZDs withdrawal). Withdrawal symptoms of Zolpidem were reported in less than $1 \%$ of subjects appearing within 48 hours of discontinuation (16). One of the probable factors associated with adverse effects of Zolpidem is gender. Women have been found to have a significantly higher serum Zolpidem concentration than men at equivalent dosage (17). Some studies demonstrated that sudden discontinuation of Zolpidem by doses within the normal recommended range 2 to 4 weeks after treatment has not been associated with withdrawal symptoms (2, 18). Zolpidem dependence and withdrawal symptoms have been reported in patients with doses between 160 to $2000 \mathrm{mg}$ per day $(7,10)$. Therefore, to our knowledge, withdrawal seizure in our case with the mentioned dosage of Zolpidem is the minimum dose reported up to now. According to other case reports and studies and our case, Zolpidem, soon after sudden discontinuation, causes withdrawal symptoms including insomnia, anxiety and epileptic attack, especially at high doses and long-term use. Concerns about Zolpidem abuse, dependence and withdrawal seizure are increasing in the recent years due to increased number of reported cases. Maybe, this event is due to unawareness of many physicians and patients about the potential of Zolpidem to create these problems. In addition, use of this drug out of its therapeutic goals and short half-life predisposes adverse events. Our case suggested that Zolpidem can potentially lead to dependence and withdrawal seizure in Iranian population, also can occur with a dosage under last reported doses. Besides, the possibility of mentioned problems cannot be excluded at any dosage. We suggest physicians to pay more attention to the potential of Zolpidem to create dependence and withdrawal seizure. Besides, they should always keep its effects in their mind and subtilize during prescription of Zolpidem for any patients and at any doses, especially for those with a previous history of drug or substance abuse and at high doses. This study presented a new dosage of Zolpidem that causes withdrawal seizure. However, this is a case study and it needs further studies to conclude about adverse effects of Zolpidem.

\section{Authors' Contributions}

Seyed Alireza Haji Seyed Javadi and Farid Hajiali were involved in acquisition of clinical data and reviewing the scientific literature. Farid Hajiali and Marjan Nassiri-Asl wrote the manuscript. All authors read and approved the final manuscript. 


\section{References}

1. Chen SC, Chen HC, Liao SC, Tseng MC, Lee MB. Detoxification of high-dose zolpidem using cross-titration with an adequate equivalent dose of diazepam. Gen Hosp Psychiatry. 2012;34(2):210 e5-7.

2. Holm KJ, Goa KL. Zolpidem: an update of its pharmacology, therapeutic efficacy and tolerability in the treatment of insomnia. Drugs. 2000;59(4):865-89.

3. Sakkas P, Psarros C, Masdrakis V, Liappas J, Christodoulou GN. Dependence on zolpidem: a case report. European Psychiatry. 1999;14(6):358-9.

4. Pourshams M, Malakouti SK. Zolpidem abuse and dependency in an elderly patient with major depressive disorder: a case report. Daru. 2014;22:54.

5. Krueger TH, Kropp S, Huber TJ. High-dose zolpidem dependence in a patient with chronic facial pain. Ann Pharmacother. 2005;39(4):773-4.

6. Wang LJ, Ree SC, Chu CL, Juang YY. Zolpidem dependence and withdrawal seizure--report of two cases. Psychiatr Danub. 2011;23(1):76-8.

7. Cubala WJ, Landowski J. Seizure following sudden zolpidem withdrawal. Prog Neuropsychopharmacol Biol Psychiatry. 2007;31(2):539-40.

8. Keuroghlian AS, Barry AS, Weiss RD. Circadian dysregulation, zolpidem dependence, and withdrawal seizure in a resident physician performing shift work. Am JAddict. 2012;21(6):576-7.

9. Victorri-Vigneau C, Dailly E, Veyrac G, Jolliet P. Evidence of zolpidem abuse and dependence: results of the French Centre for
Evaluation and Information on Pharmacodependence (CEIP) network survey. Br JClin Pharmacol. 2007;64(2):198-209.

10. Huang MC, Lin HY, Chen CH. Dependence on zolpidem. Psychiatry Clin Neurosci. 2007;61(2):207-8.

11. Salva P, Costa J. Clinical pharmacokinetics and pharmacodynamics of zolpidem. Therapeutic implications. Clin Pharmacokinet. 1995;29(3):142-53.

12. McKernan RM, Rosahl TW, Reynolds DS, Sur C, Wafford KA, Atack JR, et al. Sedative but not anxiolytic properties of benzodiazepines are mediated by the GABA(A) receptor alpha1 subtype. Nat Neurosci. 2000;3(6):587-92.

13. Courtet P, Pignay V, Castelnau D, Boulenger JP. [Abuse of and dependence on zolpidem: a report of seven cases]. Encephale. 1999;25(6):652-7.

14. Feneon D, Villemeyre Plane M, Reynaud M. Addiction au zolpidem: about a case. Alcohol Addict. 2001;23(4):519-23.

15. Liappas IA, Malitas PN, Dimopoulos NP, Gitsa OE, Liappas AI, Nikolaou Ch K, et al. Zolpidem dependence case series: possible neurobiological mechanisms and clinical management. J Psychopharmacol. 2003;17(1):131-5.

16. Toner LC, Tsambiras BM, Catalano G, Catalano MC, Cooper DS. Central nervous system side effects associated with zolpidem treatment. Clin Neuropharmacol. 2000;23(1):54-8.

17. Cubala WJ, Landowski J, Wichowicz HM. Zolpidem abuse, dependence and withdrawal syndrome: sex as susceptibility factor for adverse effects. Br J Clin Pharmacol. 2008;65(3):444-5.

18. Vartzopoulos D, Bozikas V, Phocas C, Karavatos A, Kaprinis G. Dependence on zolpidem in high dose. Int Clin Psychopharmacol. 2000;15(3):181-2. 